\title{
Modification of the Coping and the Emotional Comorbidity in Patients Candidates to Cardiac Surgery
}

\author{
Carlos Figueroa-López¹, Daniela Méndez-Rodríguez ${ }^{1}$, Yesenia Sharai Robledo-Martínez¹, \\ Bertha Ramos-Del Río ${ }^{1}$, Ana Karen Talavera-Peña ${ }^{1}$, Carlos Riera-Kinkel²* \\ ${ }^{1}$ Psychology Faculty, FES Zaragoza UNAM, México \\ ${ }^{2}$ Division Cardiothoracic Surgery, Hospital de Cardiología, Centro Médico Nacional Siglo XXI, Instituto Mexicano del Seguro \\ Social, Mexico City, Mexico \\ Email: *rierac7@gmail.com
}

How to cite this paper: Figueroa-López, C., Méndez-Rodríguez, D., Robledo-Martínez, Y. S., Ramos-Del Río, B., Talavera-Peña, A. K., \& Riera-Kinkel, C. (2018). Modification of the Coping and the Emotional Comorbidity in Patients Candidates to Cardiac Surgery. Psychology, 9, 2591-2607. https://doi.org/10.4236/psych.2018.911148

Received: September 27, 2018

Accepted: October 26, 2018

Published: October 31, 2018

Copyright $\odot 2018$ by authors and Scientific Research Publishing Inc. This work is licensed under the Creative Commons Attribution International License (CC BY 4.0).

http://creativecommons.org/licenses/by/4.0/

(c) (i) Open Access

\begin{abstract}
Objective: To evaluate the effectiveness of a program of psychological preparation for invasive procedures in patients with heart disease, candidates for cardiac surgery, to reduce emotional morbidity and improve perception of health related quality of life. Methods: A Quasi-experimental design was used. The study included a non-probabilistic randomized sample of $110 \mathrm{pa}-$ tients treated in the Division of Cardiac Surgery Centro Medico Nacional Siglo XXI Instituto Mexicano del Seguro Social IMSS. They were divided in two groups: Experimental group $(\mathrm{n}=44)$ and Control group $(\mathrm{n}=66)$. Instruments. CHIP Coping Questionnaire, HADS Anxiety and Depression Hospital Scale, Health Related Quality of Life Questionnaire and a Visual Analogue Scale (VAS) to measure postoperative pain. Results. The experimental group showed an increase in instrumental coping style compared to pre $(M=24.55)$ and post evaluation $(M=25.93)$; statistically significant differences were found in the variables of stress $(p=0.042)$, anxiety $(p=0.001)$, depression $(p=0.056)$ and health related quality of life $(p=0.000)$; while in the control group, the distraction coping style (pre $M=25.80$, post $M=$ $26.73)$ is the one that increased; there were no statistically significant differences in the variables stress $(p=0.274)$, anxiety $(p=0.671)$ and depression $(p$ $=0.850)$, except for the health related quality of life $(p=0.000)$. This study is the first in Mexico to manage emotional comorbidity in this type of population.
\end{abstract}

\section{Keywords}

Coping, Emotional Comorbidity, Stress, Anxiety, Depression, Heart Disease 


\section{Introduction}

According to Lazarus and Folkman (Lazarus \& Folkman, 1986), coping is all cognitive and behavioral effort that seeks the domain, decrease and tolerance of the demands that a stressful situation generates, such as the prospect of cardiac surgery which the perception is fear to a large incision and cognitive disturbance related with cardiopulmonary bypass machine (García-Tornel, Cañas, Hernández, Ayala, Romero, Castillo, \& Lucio, 2012; Garrido, Canovas, Cuenca, Barquero, Silva, Araji et al., 2017). Faced with this situation, the cognitive and emotional representations that the patient perceives of his illness will determine his behavior to face it, that is, the preventive behavior that the patient adopts, will be that of which he perceives greater benefits and less costs to reduce the threat of the illness (Leventhal, Nerenz, \& Steel, 1984).

The coping response of the patient can be directed towards: a) the problem, carrying out activities to modify the stressor agent, b) the emotion, performing activities to regulate the emotional responses generated by the stressor (Lazarus \& Folkman, 1986).

Endler, Parker and Summerfeldt (1998) defined four dimensions of coping response: a) Distraction, the patient uses actions and cognitions to avoid worry; b) Palliative, the response of "self-help" to relieve the discomfort of the stressor; c) Instrumental, the application of effective strategies to treat the stressor; it is about seeking professional help and learning more about their illness; and d) Emotional concern, focuses on how to resolve the emotional consequences (excessive worry) that the stressor generates.

Although all patients report the use of the four coping styles mentioned above (Figueroa-López, Mora-Osorio, \& Ramos del Rio, 2017), particularly the use of effective instrumental strategies favors cardiovascular health and better control of the disease, not so the emotional coping styles (Distraction, Palliative and Concern Emotional) because they cause greater worries towards the stressor (cardiac surgery for example) causing anxiety and depression, concern and apprehension about their future and their health, and a great autonomic hyperexcitation (Londoño \& Velasco, 2015; Vallejos \& Obergorn, 2017).

On the other hand, not only the coping styles act as an agent of psychosocial risk, there are also other psychological factors that affect the person with heart disease (Larringa \& Hernández, 2016). These factors cause not only alterations in the quality of life of these patients, they also make the occurrence of an unfavorable outcome of the surgery more likely, altering the patient's physical, emotional, cognitive and behavioral state (Borregaard, Ekholm, Riber, Sørensen, Møller, Thrysoe, \& Berg, 2018; Nekouei, Yousefy, Doost, Manshaee, \& Sadeghei, 2014). For example, psychological stress causes the sympathetic nervous system to increase blood levels of catecholamines, increasing the likelihood of myocardial ischemia and infarction (Norlund, Lissåker, Wallert, Held, \& Olsson, 2018).

Regarding the presence of anxiety and depression in these patients, a prevalence ranging from 60 to $80 \%$ has been reported (Figueroa-López, Mora-Osorio, 
\& Ramos del Rio, 2017; Vilchis, Secín, López, \& Córdova, 2010; Hweidi, Gharaibeh, Al-Obeisat, \& Al-Smadi, 2017), having consequences such as increased vulnerability to infections, longer hospital stay, a slow recovery and even more related as predictors of post surgical pain (Sandrino, Meléndez, Mederosm, \& Carracedo, 2016; Hernández, López, \& Guzmán, 2016).

Another factor recognized as risk is the health related quality of life. This is affected by the presence of clinical symptoms (decreased breathing, muscle fatigue, chest pain, dizziness) and emotional (such as anxiety, feelings of sadness, fear, depression and stress as a result of the perception of the disease). The perception of their health, occupational functioning, and carrying out physical and social activities are also altered, negatively affecting the recovery of their health (Figueroa-López, Mora-Osorio, \& Ramos del Rio, 2017; Achury, Rodríguez, Agudelo, Hoyos, \& Acuña, 2011; Barrantes, 2010).

There are some psychological variables that predict and modify postoperative pain, such as anxiety and depression, which cause in the patient respiratory complications, higher doses of analgesia and morphine, increased hospital stay and long-term chronic pain causing a minor recovery daily activities and possible hospital readmissions (Gallardo, 2010; Gómez, Monsalve, Soriano, \& De Andrés, 2007; Muñoz, Salmerón, Santiago, \& Marcote, 2001).

Currently, cognitive behavioral therapy is the most commonly used in the evaluation and psychological treatment of psychosocial risk factors in patients who are going to undergo cardiac surgery, since they focus on the prevention of the disease, changes in lifestyle, to control emotions and rehabilitation of patients through the change of cognitions and behavior, to provide the pre-surgical patient information about their illness and treatment, stimulating emotional expression, establishing relationships of trust between the patient and staff of the hospital, to control levels of depression and anxiety, and the acquisition of healthy behaviors to modify habits of cardiovascular risk (Sánchez-Román, Velasco-Furlong, Rodríguez-Carvajal, \& Baranda-Sepúlveda, 2006; Castillero, 2007). Among the main techniques of psychological intervention are relaxation, psycho-education of the disease, guided visualization and problem solving (Figueroa-López, Mora-Osorio, \& Ramos del Rio, 2017; Londoño \& Velasco, 2015; Vallejos \& Obergorn, 2017).

In this sense, Bringas et al. (2006), confirmed the efficacy of this type of program in patients who are candidates for surgery to reduce levels of anxiety and depression using emotional expression, breathing, relaxation and guided visualization. Likewise, Miguel (2016) and López (2016) with patients for cardiac surgery successfully used the psycho-education technique to modify emotional comorbidity and perception of control of the disease.

In summary, living with a heart disease and facing the stressful experience of the perspective of being operated can disturb the patient's life and lead to negative psychological consequences such as depression, anxiety and decreased quality of life. 
Identifying the coping styles that patients use when dealing with stressors in the hospital environment could help develop strategies to improve psychological distress.

In Mexico, evaluation and psychological intervention protocols have not been implemented to evaluate these psychological variables in patients candidates for cardiac surgery.

OBJECTIVE

To evaluate the effectiveness of a psychological preparation program in cardiac patients tributary to invasive procedures to modify the coping styles, decrease the emotional comorbidity and improve the perception of health related quality of life of these patients.

\section{Method}

This study was held At the Cardiology Hospital. Centro Médico Nacional Siglo XXI. Instituto Mexicano del Seguro Social Siglo XXI. Mexico City, from July 2017 to May 2018.

It was used a quasi-experimental design, including a pre and post test phase with control group (Montero \& León, 2005).

Participants. Randomized non-probabilistic sample of patients who were tributary to cardiac surgery. They were randomly assigned to the Treatment Group (G1) and the Control Group (G2). Men and women were included. Patients were not included in the study if they had chronic health problems at an advanced stage (renal failure, COPD, etc.), nor those who at the time of the study were taking anxiolytics and antidepressants.

Independent Variable. Psychological preparation for invasive procedures: cognitive-behavioral psychological strategies focused on the reduction of anxiety and psychological stress associated with invasive and surgical medical procedures (Rodríguez, 2000).

\section{Dependent variables.}

Coping: constantly changing cognitive and behavioral efforts develop to handle specific external or internal demands that are overflow the individual's resources (Lazarus \& Folkman, 1986). It was measured using the Inventory of Coping with Injuries and Health Problems (CHIP).

Anxiety: emotional reaction involved in the processes of adaptation to real or imaginary adverse events, which prepares the body to react to that situation (Crandall, Halterman, \& Mckner, 2007). It was measured using the Hospital Anxiety and Depression Scale (HADS).

Depression: mood disorder with feelings of sadness, low self-esteem, pessimistic attitude, loss, anger, or frustration interfere with everyday life of the individual over a period of time (Snaith, 2003). It was measured using the Hospital Anxiety and Depression Scale (HADS).

Health related quality of life: personal perception of the impact of illness or treatment about feelings of well-being and the ability to do personal activities 
(Fernández, Hernández, \& Siegrist, 2001). It was measured using the Health Related Quality of Life.

Post-surgical pain: acute pain that appears as a consequence of the surgical act (Soler, Faus, Montaner, Faus, Morales, \& Martínez-Pons, 1999). It was measured using the Visual Analogue Scale (VAS).

\section{Questionnaires}

Clinical History of Health and Stress. Clinical information of these patients and data on stress symptoms.

Inventory of Coping with Injuries and Health Problems (CHIP) (Endler, Parker, \& Summerfeldt, 1998). Consists of four scales: Distraction, Palliative, Instrumental, Emotional preoccupation.

Hospital Anxiety and Depression Scale (HADS) (Zigmond \& Snaith, 1983). It consists of 14 reagents in two subscales of seven reagents each. It is a Likert scale with four response options.

Health Related Quality of Life (Ramos, Figueroa, Alcocer, \& Rincon, 2011). Consists of 23 items with a Likert scale, evaluation to the following dimensions: physical limitations, symptoms, quality of life, social interference, self-efficacy, functional status and clinical summary.

Visual Analogue Scale (VAS): to measure postoperative pain (Gómez, Gómez, \& Mirabete, 2016).

\section{Procedure}

It consisted of three stages: I. Pre-evaluation, II. Psychological preparation, III. Post-evaluation. Five individual sessions were held, each lasting 60 minutes.

Stage I and III. A Psychological Assessment was performed in two sessions (sessions 1 and 5), which included the identification card, Clinical History of Health and Stress and the evaluation of coping styles, anxiety, depression, quality of life variables and the VAS to measure post-surgical pain only in the post-evaluation.

Stage II (Psychological Preparation). It was based on psychoeducation and cognitive behavioral strategies to reduce emotional comorbidity. It was carried out in three sessions (2, 3 and 4).

Ethical considerations. Patients were informed about their right to participate signing an informed consent form. The ethical considerations of this protocol were based on the Helsinki Declaration of the World Medical Association (2008).

\section{Results}

The data were analyzed using the SPSS v.22. Of the evaluated patients, $66(60 \%)$ were assigned to the control group and 44 (40\%) to the experimental group; 110 patients candidates for cardiac surgery were evaluated, 55 (61\%) of valve replacement, $29(32 \%)$ of coronary revascularization and six (7\%) with diagnosis of interauricular communication; 57 men (52\%), and 53 women (47\%) with a 
mean age of 62 years and a range of 19 to 81 years . $67 \%$ were married; with a high school level or technical career (31\%). 35\% were dedicated to the home and $87 \%$ were Catholics (see Table 1).

Table 1. Sociodemographic data.

\begin{tabular}{|c|c|c|c|c|}
\hline & \multicolumn{2}{|c|}{ Control Group } & \multicolumn{2}{|c|}{ Experimental Group } \\
\hline & $N=44$ & $\%$ & $N=66$ & $\%$ \\
\hline \multicolumn{5}{|l|}{ Sex } \\
\hline Male & 33 & 50 & 24 & 55 \\
\hline Female & 33 & 50 & 20 & 45 \\
\hline \multicolumn{5}{|l|}{ Procedence } \\
\hline Local & 44 & 67 & 38 & 86 \\
\hline Foreign & 22 & 33 & 6 & 14 \\
\hline \multicolumn{5}{|l|}{ Civil status } \\
\hline Single & 11 & 17 & 5 & 11 \\
\hline Married & 41 & 62 & 33 & 75 \\
\hline Widower & 12 & 18 & 3 & 7 \\
\hline Divorced & 2 & 3 & 3 & 7 \\
\hline \multicolumn{5}{|l|}{ Scholarship } \\
\hline Illiterate & 2 & 3 & 0 & 0 \\
\hline Read and write & 6 & 9 & 1 & 2 \\
\hline Primary & 12 & 18 & 9 & 20 \\
\hline High school & 7 & 11 & 8 & 18 \\
\hline Technique & 19 & 29 & 15 & 3.4 \\
\hline Bachelor's degree & 19 & 29 & 8 & 18 \\
\hline Postgraduate & 1 & 1 & 3 & 7 \\
\hline \multicolumn{5}{|l|}{ Occupation } \\
\hline Home & 26 & 39 & 12 & 27 \\
\hline Employee & 11 & 17 & 10 & 2.3 \\
\hline Merchant & 5 & 8 & 2 & 5 \\
\hline Professional & 7 & 11 & 8 & 18 \\
\hline Retired & 16 & 24 & 11 & 25 \\
\hline Students & 1 & 1 & 1 & 2 \\
\hline \multicolumn{5}{|l|}{ Religion } \\
\hline None & 2 & 3 & 2 & 4 \\
\hline Catholic & 58 & 88 & 38 & 87 \\
\hline Christian & 4 & 6 & 3 & 7 \\
\hline Other & 2 & 3 & 2 & 4 \\
\hline
\end{tabular}


The reliability analysis of the CHIP inventory reports an adequate Cronbach's alpha of 0.77 . The results obtained from the pre-evaluation show that the coping strategy most used by the patients in the control group was distraction ( $\mathrm{X}=$ 25.8), that is, they prefer to do activities such as thinking about better times, being with other people, listening to music, between others, followed by instrumental strategy $(X=25.6)$, then palliative $(X=24.9)$ and emotional was the least used by patients $(X=17.7)$. In the experimental group, the most used strategy was Instrumental $(\mathrm{X}=24.6)$, that is, they prefer to obtain more information, seek treatment immediately, focus on their recovery, followed by the distraction strategy $(X=24.3)$, then the palliative $(X=24.1)$ and emotional was the least used by patients $(\mathrm{X}=18.5)$ (see Table 2$)$.

In the post-evaluation it is shown that the coping strategy most used by the patients in the control group continues to be the distraction $(X=26.7)$, followed by the instrumental strategy $(X=25.9)$, then the palliative strategy $(X=25.3)$ and the emotional one less used $(X=17.1)$. In the experimental group, the most used coping strategy is still the Instrumental $(X=25.9)$, followed by the palliative strategy $(X=25.6)$, then the Distraction $(X=25.5)$ and emotional was the least used by the patients $(\mathrm{X}=16.5)$. A t-test for related samples was performed to measure the significance of the differences in the use of each coping strategy between pre- and post-evaluation, only statistically significant differences were found for the palliative strategy in the experimental group $(p=0.019)$. In the distraction strategy, no statistically significant differences were found for the control group ( $p=0.425)$ or experimental group $(p=241)$; nor for the palliative strategy in the control group $(p=0.796)$; neither in the instrumental strategy nor for the control group $(p=0.218)$ nor for the experimental one $(p=0.909)$; Likewise, no statistically significant differences were found for the emotional strategy neither for the control group $(p=0.539)$ nor for the experimental group ( $p=$ 0.731) (see Table 2).

According to the reliability analysis, the stress symptoms questionnaire reported an adequate Cronbach's alpha of 0.84 . The results showed that in the control group only $9 \%$ presented moderate levels of stress and in the experimental group $25 \%$ of the sample presented a moderate or high level of stress symptoms before the operation (pre-evaluation). In the post-evaluation the

Table 2. Coping with injuries and health problems (CHIP).

\begin{tabular}{ccccccccc}
\hline & \multicolumn{3}{c}{ Pre-evaluation } & \multicolumn{3}{c}{ Post-evaluation } \\
\cline { 2 - 8 } Style & \multicolumn{2}{c}{ Control } & \multicolumn{2}{c}{ Experimental } & \multicolumn{2}{c}{ Control } & Experimental \\
\cline { 2 - 8 } & $\mathrm{X}$ & $\mathrm{SD}$ & $\mathrm{X}$ & $\mathrm{SD}$ & $\mathrm{X}$ & $\mathrm{SD}$ & $\mathrm{X}$ & $\mathrm{SD}$ \\
\hline Distraction & 25.8 & 3.6 & 24.3 & 4.1 & 26.7 & 3.0 & 25.5 & 3.0 \\
Palliative & 24.9 & 4.0 & 24.1 & 3.4 & 25.3 & 2.8 & 25.5 & 3.3 \\
Instrumental & 25.6 & 4.8 & 24.6 & 4.4 & 25.9 & 3.4 & 25.9 & 3.9 \\
Emotional & 17.7 & 4.7 & 18.5 & 5.4 & 17.1 & 3.8 & 16.5 & 4.6 \\
\hline
\end{tabular}


control group increased the number of patients with stress symptoms (27\%) while in the experimental group it decreased (9\%). A $\mathbf{t}$ test was performed for related samples in which statistically significant differences were found between the pre and post evaluation of the experimental group $(p=0.042)$, but not for the control group $(p=0.274)$ where no statistically significant differences were found (see Table 3).

The Hospital Anxiety and Depression scale showed an adequate Cronbach alpha of 0.86 . In the Anxiety scale, $54 \%$ of the patients in the control group presented symptoms of anxiety (from mild to severe) before undergoing surgery, and in the post-evaluation it decreased to $34 \%$. On the other hand, in the pre-evaluation, $64 \%$ of the patients in the experimental group reported anxiety (moderate to severe), while in the post-evaluation it decreased to $39 \%$. A $t$ test was performed for related samples in which statistically significant differences were found between the pre and post evaluation of the experimental group ( $p=$ 0.001 ), but not for the control group ( $p=0.671$ ) where no statistically significant differences were found (see Table 4).

In the pre-evaluation, the control group reported $50 \%$ while the experimental group $57 \%$ of some degree of depression (Moderate or severe). In more detail, the control group showed a $38 \%$ moderate depression, which increased to $47 \%$ in the posttest. The same happened in the severe one, which increased from $12 \%$ to $13 \%$. On the other hand, in the experimental group, severe depression decreased from $25 \%$ in the pretest to $7 \%$ in the posttest, increasing in this sense moderate depression from $32 \%$ to $52 \%$. In the $\mathrm{t}$-test for related samples, a level of borderline significance was obtained for the differences between the pre and post evaluation of the experimental group ( $p=0.056)$, but not for the control group $(p=0.850)$ where no statistically differences were found significant (see Table 5 ).

Table 3. Stress symptoms.

\begin{tabular}{ccccccccc}
\hline & \multicolumn{3}{c}{ Pre-evaluation } & \multicolumn{3}{c}{ Post-evaluation } \\
\cline { 2 - 9 } Level & \multicolumn{2}{c}{ Control } & \multicolumn{2}{c}{ Experimental } & \multicolumn{2}{c}{ Control } & \multicolumn{2}{c}{ Experimental } \\
\cline { 2 - 9 } & $\mathrm{N}$ & $\%$ & $\mathrm{~N}$ & $\%$ & $\mathrm{~N}$ & $\%$ & $\mathrm{~N}$ & $\%$ \\
\hline Low & 60 & 91 & 33 & 75 & 48 & 73 & 40 & 91 \\
Moderate & 6 & 9 & 10 & 2.3 & 18 & 27 & 4 & 9 \\
High & 0 & 0 & 1 & 2 & 0 & 0 & 0 & 0 \\
\hline
\end{tabular}

Table 4. Hospital anxiety scale (HADS).

\begin{tabular}{ccccccccc}
\hline & \multicolumn{3}{c}{ Pre-evaluation } & \multicolumn{3}{c}{ Post-evaluation } \\
\cline { 2 - 9 } Level & \multicolumn{2}{c}{ Control } & \multicolumn{2}{c}{ Experimental } & \multicolumn{2}{c}{ Control } & \multicolumn{2}{c}{ Experimental } \\
\cline { 2 - 9 } & $\mathrm{N}$ & $\%$ & $\mathrm{~N}$ & $\%$ & $\mathrm{~N}$ & $\%$ & $\mathrm{~N}$ & $\%$ \\
\hline None & 30 & 46 & 16 & 36 & 44 & 66 & 27 & 61 \\
Moderate & 29 & 44 & 18 & 41 & 18 & 27 & 13 & 30 \\
Severe & 7 & 10 & 10 & 23 & 2 & 7 & 4 & 9 \\
\hline
\end{tabular}


The reliability analysis of the quality of life related to health presents an adequate Cronbach's alpha of 0.92. The descriptive analysis of the pre-evaluation in the control group showed that patients regularly perceive their well-being and physical satisfaction, reflected in the Life Quality score $(X=54.6)$ and Clinical Summary $(X=67.0)$. In the Functional State, patients obtained an average of 71.3 , which reflects the impact regular perception of patients in matters of their physical functioning, as well as the impact of the symptoms (see Table 6).

In the experimental group patients showed a regular level regarding how they perceive their well being and physical satisfaction scores reflecting Quality Life $(\mathrm{X}=61.8)$ and Clinical Summary $(\mathrm{X}=67.8)$. In the Functional State, patients obtained an average of 69.8 , which reflects a greater impact that patients perceive in matters of their physical functioning, as well as a moderate impact of the symptoms (see Table 6).

The descriptive analysis of the post-evaluation in the control group showed that patients improved their perception of well-being and physical satisfaction, reflected in the Life Quality score $(X=82.7)$ and Clinical Summary $(X=89.0)$. In the Functional State, patients obtained an average of 90.0, which reflects the perception of patients in matters of their physical functioning, as well as the

Table 5. Hospital depression scale (HADS).

\begin{tabular}{ccccccccc}
\hline & \multicolumn{4}{c}{ Pre-evaluation } & \multicolumn{3}{c}{ Post-evaluation } \\
\cline { 2 - 8 } Level & \multicolumn{2}{c}{ Control } & \multicolumn{2}{c}{ Experimental } & \multicolumn{2}{c}{ Control } & Experimental \\
\cline { 2 - 8 } & $\mathrm{N}$ & $\%$ & $\mathrm{~N}$ & $\%$ & $\mathrm{~N}$ & $\%$ & $\mathrm{~N}$ & $\%$ \\
\hline None & 33 & 50 & 19 & 43 & 26 & 40 & 18 & 41 \\
Moderate & 25 & 38 & 14 & 32 & 31 & 47 & 2.3 & 52 \\
Severe & 8 & 12 & 11 & 25 & 7 & 13 & 3 & 7 \\
\hline
\end{tabular}

Table 6. Health-related quality of life (HRQoL).

\begin{tabular}{ccccccccc}
\hline & \multicolumn{3}{c}{ Pre-evaluation } & \multicolumn{5}{c}{ Post-evaluation } \\
\cline { 2 - 9 } & \multicolumn{2}{c}{ Control } & Experimental & Control & Experimental \\
\cline { 2 - 9 } & $\mathrm{X}$ & $\mathrm{SD}$ & $\mathrm{X}$ & $\mathrm{SD}$ & $\mathrm{X}$ & $\mathrm{SD}$ & $\mathrm{X}$ & $\mathrm{SD}$ \\
\hline Physical limitations & 63.8 & 18.9 & 65.8 & 17.5 & 98.9 & 5.2 & 100 & 5.2 \\
Symptom & 77.3 & 22.5 & 72.2 & 22.4 & 80.5 & 19.9 & 83.9 & 19.9 \\
Symptom frequency & 83.3 & 21.7 & 75.3 & 25.4 & 81.8 & 22.9 & 88.9 & 22.9 \\
Symptom severity & 76.1 & 22.9 & 74.4 & 20.4 & 80.1 & 17.7 & 78.6 & 17.7 \\
Symptom evolution & 62.2 & 37.3 & 59.5 & 37.0 & 74.4 & 30.8 & 82.6 & 30.8 \\
Self-efficacy & 63.6 & 24.4 & 70.8 & 16.9 & 67.4 & 19.6 & 71.0 & 19.6 \\
Quality of life & 54.6 & 22.0 & 61.8 & 18.4 & 82.7 & 12.6 & 81.8 & 12.6 \\
Social interference & 61.0 & 31.4 & 67.0 & 29.0 & 99.4 & 3.3 & 99.1 & 3.3 \\
Functional state & 71.3 & 18.0 & 69.8 & 18.0 & 90.0 & 9.3 & 92.5 & 9.3 \\
Clinical summary & 67.0 & 15.8 & 67.8 & 16.8 & 89.0 & 8.3 & 91.0 & 8.3 \\
\hline
\end{tabular}


impact of the symptoms (see Table 6).

In the experimental group patients improved perception of well being and physical satisfaction reflected in increased life quality score $(\mathrm{X}=81.81)$ and Clinical Summary ( $\mathrm{X}=$ January 9.0). In the Functional State, the patients obtained an average of 92.5 , which reflects that they improved their perception in matters of their physical functioning, as well as the impact of the symptoms. A $\mathbf{t}$ test was performed for related samples in which statistically significant differences were found between the pre and post evaluation for both the experimental group $(p=0.000)$ and the control group $(p=0.000)$ (see Table 6).

The pain scale showed that in the control group $40 \%$ of patients had a slight level of pain and $17 \%$ non sint tio any pain. While in the experimental group $43 \%$ of patients reported an average pain and $13 \%$ did not report pain. A t-test was performed for independent samples and no statistically significant differences were found in the evaluation of pain between the control and experimental groups $(p=0.237)$ (see Table 7).

\section{Discussion}

The objective of the present investigation was to evaluate the effectiveness of a psychological preparation program for invasive procedures in patients with high-risk cardiac surgery to modify their coping styles, decrease emotional comorbidity and improve the perception of health related quality of life.

Regarding coping strategies, significant clinical differences were found in both groups after the intervention program. From the initial evaluation, the experimental group reported the instrumental strategy as the most reported, and the posttest increased its use, that is, the patients do everything to focus on the search for quick and direct information about their treatment and recovery. In contrast, the control group did not change the coping strategies reported, the most used both in pretest and posttest was the distraction, which in relation to their illness, means that patients prefer to occupy their time with family or friends, or watch TV or listen to music. Knowing that psychological stress is a psychosocial cardiovascular risk factor, its potential impact on the health of the individual can be directly related to the way in which stress is being dealt with.

Regarding the fact that the symptoms of stress would decrease with respect to

Table 7. Post-operative pain.

\begin{tabular}{ccccc}
\hline & \multicolumn{2}{c}{ Control group } & \multicolumn{2}{c}{ Experimental group } \\
\cline { 2 - 5 } & N & $\%$ & N & $\%$ \\
\hline No pain & 10 & 17 & 6 & 13 \\
Soft pain & 26 & 40 & 50 & 35 \\
Moderate pain & 17 & 26 & 19 & 43 \\
Severe pain & 7 & 11 & 4 & 9 \\
Worst possible pain & 4 & 6 & 0 & 0 \\
\hline
\end{tabular}


the initial values in the experimental group more than in the control group, statistical and clinical differences in favor of the first group can be observed. This result is very important since it is known that psychological stress affects the heart through the autonomic nervous system. Specifically, the expression of symptoms in the face of acute stress, such as the expectation of a high-risk surgery, causes the sympathetic nervous system to generate the "fight or flight" response characterized by elevation of blood pressure, heart rate, glucose, cortisol, adrenaline. Similarly, it has also been associated with increased cardiovascular reactivity and a decrease in the variability of heart rate, among other responses. Through several studies, it has been identified that stress is a common characteristic in patients close to surgery (Miguel, 2016; López, 2016; Escudero, 2016).

During the preoperative period, high levels of stress can cause alterations in the physiological, emotional, cognitive and behavioral plane. In addition, patients tend to express greater pain or suffering, more concerns about the subject of the application of anesthesia and complications of the latter, can result in disability or death (Sobieralska, Rydygier, Copernicus, Copernicus, Michalak, Wielki, et al., 2015). Escamilla (2018) documented that stress can also cause alterations in the response of the organism, delaying the healing process, weakening the immune system and associating with a prolonged postoperative hospital stay, due to the mechanism of biological over-activation, which is why it is considered an additional risk factor that negatively affects postoperative recovery (Pérez, 2007). In addition to is to, patients often behave aggressively, negative attitudes towards taking care of her illness, besides having problems with health workers and raising excessively analgesic consumption.

Regarding that the emotional comorbidity would be modified, the posttest results show a clinical and statistically significant decrease $(p=0.001)$ of the anxiety in the experimental group compared to the control group, who did not show significant changes. These results imply that patients now report less transient fear, uncertainty and apprehension for the management of their heart disease. In addition, it has been proven that anxiety is an extremely influential factor for the physical and emotional recovery of patients, since it is considered an adaptive psychological disposition that allows facing the intervention if it presents with a mild symptomatology. However, high levels of anxiety are associated with psychological and physical discomfort, which brings with it various complications when the symptoms are moderate to severe (Cárdenas, Quiroga, Restrepo, \& Cortés, 2005; De La Paz, Prego, \& Barzaga, 2006; Bradt, Dileo, \& Shim, 2013; Figueroa, Alcocer, \& Ramos, 2016).

Regarding depression, as has been reported in other studies (Pirraglia, Peterson, Williams, Gorki, \& Charlson, 1999; Carneiro, Telles, Rassi, Siquiera et al., 2009; Patron, Messerotti, \& Palomba, 2014), there is a high prevalence of this variable in patients with heart disease. Again, the effect of the intervention program is observed in the results since the experimental group significantly de- 
creased the report of depression from a severe to a mild level compared to the control group, which increased both in the mild and severe levels. This result is important since cardiac patients with depression have shown a low adherence to physical activity, and to the intake of medications, and it has been related to inflammatory processes and dysfunction in the autonomic nervous system (García-Tornel, Cañas, Hernández, Ayala, Romero, Castillo, \& Lucio, 2012; Figueroa, Alcocer, \& Ramos, 2016; Garrido, Canovas, Cuenca, Barquero, Silva, Araji et al., 2017).

On the other hand, although it has been reported that due to anticipatory stress and the immediate result of surgery, up to $20 \%$ of patients show no improvement in health-related quality of life after cardiac surgery despite the procedures Surgical successes (Rothenhäusler, Ehrentraut, Kapfhammer, Lang, Zachoval, Bilzer, Schelling, \& Gerbes, 2002), in this study both groups clinically and statistically improved their perception of disease symptoms from an average score in the pretest to adequate in the posttest. The patient's perception of the symptoms of his disease affects the way in which he is involved in reaching his personal and social goals, expectations and interests, therefore he becomes a predictive variable of the response to treatment. In this sense, the evaluation of quality of life becomes a very useful indicator in the evaluations of health interventions in the management of cardiovascular risk (Lemos, Agudelo, Arango, \& Rogers, 2013).

Regarding postoperative pain, the experimental group reported less pain in the "severe" category (9\%) compared to the control group (11\%). The patient understands that if he has control over pain, stress decreases, his quality of life improves, and he works better.

It is important to note that psychological stress, anxiety, depression, quality of life related to health, postoperative pain and cognitive and behavioral coping strategies can change over time with psychological techniques, which can potentially have a positive impact by improving lifestyle and promoting emotional well-being. These types of patients tend to show a better emotional fit, are more proactive in seeking information related to their illness and make better decisions. Even some studies have shown that they improve the therapeutic adherence towards pharmacological treatment (Escudero, 2016).

The cognitive-behavioral techniques used in this research prove to be effective ways to help patients modify emotional comorbidity, mainly in high-risk patients such as those in this clinical population. The psychoeducational program aimed at improving knowledge of the disease, breathing techniques, relaxation and problem solving has empowered patients to cope better with their disease. For example, the systematic practice of relaxation as a technique for stress management not only reduces the physical symptomatology related to stress but also reduces certain negative emotions, for example, depression (Rozanski, Blumenthal, Davidson, Saab, \& Kubzansky, 2005; Figueroa, Alcocer, \& Ramos, 2016).

The learning that is promoted from this approach is progressive rather than 
rapid, tends to strengthen self-efficacy as patients understand the relationship between their behavior and health, and ensures that patients commit themselves to generating their own behavioral changes by identifying the risk factors that they wish to change and/or control. The program developed in this work proves to be a viable, reproducible, reliable and ideally affordable and "friendly" psychological evaluation and intervention option.

Some limitations of this study is that although it is known that coping is dynamic, in this research it was evaluated transversally. There was also no analysis by groups by level of education, by religion, or by gender, or the evaluation of social support, which can influence the results. For example, patients who perceive a strong social support might differ in the the way they cope the cardiac surgery (Alcedo \& Angulo, 2015).

Finally, a strength of this study is that it is the first in Mexico to handle coping with cardiac surgery and emotional comorbidity in this type of population. This study makes it clear that medical professionals must take time and assume as their responsibility the incorporation of the psychologist to develop evaluation and intervention programs of the emotional state of the patients. Doctors should not ignore the patient's emotional state assuming that he will only take charge of his situation. But anxiety and depression will not disappear just by ignoring them. The psychological and physiological expressions of the emotional burden in themselves can be disastrous for the psychological balance of the patient or can lead to behaviors that unnecessarily impede recovery when apparently surgery or medical treatment have been successful.

\section{Conclusion}

This study proved the effectiveness of a psychological preparation program for patients candidate to cardiac surgery, to reduce psychological distress and improve emotional perception of health related quality of life. Clinical differences and statistics were found in coping, anxiety, depression and quality of life in favor of the experimental group. The patients tend to show better emotional adjustment, are more proactive when searching for information related to their illness and make better decisions about their cardiovascular condition.

\section{Funding}

This study was supported by PAPIIT IN 304717, DGAPA, UNAM.

\section{Conflicts of Interest}

The authors declare no conflicts of interest regarding the publication of this paper.

\section{References}

Achury, D., Rodríguez, S. M., Agudelo, L. A., Hoyos, J. R., \& Acuña, J. A. (2011). Quality of Life of the Patient with Cardiovascular Disease Attending the Cardiac Rehabilitation 
Program. Nursing Research: Image and Development, 13, 49-74.

Alcedo, J., \& Angulo, J. (2015). Protective Factors, Healthy Lifestyles and Cardiovascular Risk. Psychology and Health, 25, 57-71.

Barrantes, M. (2010). Factors Associated with Quality of Life Related to Health in Patients with Coronary Heart Disease and Diabetes Mellitus. Medical Journal Herediana, 21.

Borregaard, B., Ekholm, O., Riber, L., Sørensen, J., Møller, J. E., Thrysoe, L., \& Berg, S. K. (2018). Patient-Reported Outcomes after Aortic and Mitral Valve Surgery-Results from the DenHeart Study. European Journal of Cardiovascular Nursing, 17, 246-254. https://doi.org/10.1177/1474515117731391

Bradt, J., Dileo, C., \& Shim, M. (2013). Interventions with Music for Preoperative Anxiety. Cochrane Database of Systematic Reviews, 6. https://doi.org/10.1002/14651858.CD006908.pub2

Bringas, S., Krikorian, A., Hernando, F., Rodríguez, O., Jarabo, J. R., Fernández, E., \& Balibrea, J. L. (2006). Protocol of Psychosocial Intervention in Cancer Patients Undergoing Thoracic Surgery. Psycho-Oncology, 3, 407.

Cárdenas, A., Quiroga, C., Restrepo, M., \& Cortés, D. (2005). Hysterectomy and Anxiety: Comparative Study between Two Types of Pre-Surgical Psychological Preparation. Colombian Journal of Obstetrics and Gynecology, 56, 209-215.

Carneiro, A., Telles, L., Rassi, A., Siquiera, N. et al. (2009). Evaluation of Preparatory Anxiety and Depression in Patients Undergoing Invasive Cardiac Procedures. Brazilian Journal of Anesthesiology, 59, 431-438. https://doi.org/10.1590/S0034-70942009000400005

Castillero, A. Y. (2007). Psychological Intervention in Heart Care Surgery. Advances in Latin American Psychology, 25, 52-63.

Crandall, W. V., Halterman, T. E., \& Mckner, L. M. (2007). Anxiety and Pain Symptoms in Children with Inflammatory Bowel Disease and Functional Gastrointestinal Disorders Ornder Going Colonoscopy. Journal of Pediatric Gastroenterology and Nutrition, 44, 63-67. https://doi.org/10.1097/01.mpg.0000239733.79487.1e

De La Paz, C., Prego, C., \& Barzaga, E. (2006). Fear and Anxiety at Anesthesia in Patients Undergoing Surgery. Anesthesiology Mexican Journal, 29, 159-162.

Endler, N. S., Parker, J. D., \& Summerfeldt, L. J. (1998). Coping with Health Problems: Developing a Reliable and Valid Multidimensional Measure. Psychological Assessment Journal, 10, 195.

Escamilla, M. (2018). Preoperative Stress and Anxiety Can Delay Recovery. http://www.susmedicos.com/art_estres_preoperatorio.htm

Escudero, R. B. (2016). Psychological Profile of the Hospitalized Patient Who Is a Candidate for Coronary Revascularization Surgery. Bachelor Thesis, Mexico City: UNAM.

Fernández, J. A., Hernández, R., \& Siegrist, J. (2001). The Quality of Life Profile for Chronic Patients (PECVEC): A Method to Assess Well-Being and Functionalism in Clinical Practice. Primary Care, 1, 680-689.

Figueroa-López, C., Alcocer Díaz-Barreiro, L., \& Ramos Del Rio, B. (2016). Psychological Intervention to Modify Anxiety, Depression and Quality of Life in Patients with an Implantable Pacemaker. Psychology, 7, 373-381.

Figueroa-López, C., Mora-Osorio, R., \& Ramos del Rio, B. (2017). Relationship between Preoperative Anxiety and Coping Strategies in Hospitalized Patients with Ischemic Heart Disease Candidates for Cardiac Bypass Slopes. Specialized Magazine in Health Sciences, 20, 5-9.

Gallardo, J. (2010). Postoperative Pain: Past, Present and Future. Revista Chilena de 
Anestesia, 39, 69-75.

García-Tornel, M. J., Cañas, A. C., Hernández, T. C., Ayala, J. M. C., Romero, J. M. C., Castillo, J. J. C., \& Lucio, C. A. M. (2012). Cardiovascular Surgery. Definition, Organization, Activity, Standards and Recommendations. Cardiovascular Surgery, 19, 15-38.

Garrido, J. M., Canovas, S., Cuenca, J., Barquero, J. M., Silva, J., Araji, O. et al. (2017). Comprehensive Approach to Aortic Valve Disease. Transcatheter Aortic Valve Implant. Position and Recommendations of the Spanish Society of Thoracic and Cardiovascular Surgery. Cardiovascular Surgery, 25, 102-111.

Gómez, P. M., Gómez, P. C., \& Mirabete, R. I. (2016). Creation and Validation of an Instrument for the Assessment of Cardiac Ischemic Pain. Nursing in Cardiology, 23, 45-52.

Gómez, P., Monsalve, V., Soriano, J. F., \& De Andrés, J. (2007). Emotional Alterations and Psychological Needs of Patients in an Intensive Care Unit. Intensive Care Medicine, 31, 318-325.

Hernández, A. I., López, A. J., \& Guzmán, J. A. (2016). Level of Anxiety and Preoperative Information in Patients Scheduled for Surgery. A Descriptive Cross-Sectional Study. Medical Record Group Angeles, 14, 6-11.

Hweidi, I. M., Gharaibeh, B. A., Al-Obeisat, S. M., \& Al-Smadi, A. M. (2017). Prevalence of Depression and Its Associated Factors in Patients Post-Coronary Artery Bypass Graft Surgery. Journal of Research in Nursing, 23, 76-88. https://doi.org/10.1177/1744987117728314

Larringa, V., \& Hernández, E. (2016). Psychosocial Factors Related to Cardiovascular Diseases and Cardiac Surgery. Missing Magazine, 22.

Lazarus, R. S., \& Folkman, S. (1986). Stress and Cognitive Processes. Barcelona: Martínez Roca.

Lemos, M., Agudelo, D., Arango, J., \& Rogers, H. (2013). Quality of Life in Patients with Cardiovascular Disease: Analysis of Trajectory by Sex and Depressive Symptoms. National Journal of Public Health, 31, 110-116.

Leventhal, H., Nerenz, D. R., \& Steel, D. S. (1984). Illness Representations and Coping with Health Threats. In A. Baum, S. E. Taylor, \& J. E. Singer (Eds.), Handbook of Psychology and Health, Volume IV: Social Psychological Aspects of Health (pp. 219-252). Hillsdale, NJ: Erlbaum.

Londoño, C., \& Velasco, M. (2015). Coping Style, Dispositional Optimism, Depression, Body Image, BMI and Risk of Eating Disorders as Predictors of Quality of Life Related to Health. Psychology: Advances in the Discipline, 9, 35-47.

López, J. M. (2016). Intervention Problem Solving to Reduce Emotional Comorbidity in Post-Operated Patients of Coronary Revascularization. Bachelor Thesis, Mexico City: UNAM.

Miguel, J. (2016). Efficacy of Psychoeducation to Modify Anxiety, Depression and Perception of Control in Hospitalized Patients with Ischemic Heart Disease. Bachelor Thesis, Mexico City: UNAM.

Montero, I., \& León, O. (2005). Classification System of the Method in the Research Reports in Psychology. International Journal of Clinical and Health Psychology, 5, 115-127.

Muñoz, F., Salmerón, J., Santiago, J., \& Marcote, C. (2001). Complications of Postoperative Pain. Revista de la Sociedad Española del Dolor, 8, 194-211.

Nekouei, Z. K., Yousefy, A., Doost, H. T. N., Manshaee, G., \& Sadeghei, M. (2014). Structural Model of Psychological Risk and Protective Factors Affecting Quality of Life 
in Patients with Coronary Heart Disease: A Psychocardiology Model. Journal of Research in Medical Sciences, 19, 90.

Norlund, F., Lissåker, C., Wallert, J., Held, C., \& Olsson, E. M. (2018). Factors Associated with Emotional Distress in Patients with Myocardial Infarction: Results from the SWEDEHEART Registry. European Journal of Preventive Cardiology, 25, 910-920. https://doi.org/10.1177/2047487318770510

Patron, E., Messerotti, S., \& Palomba, D. (2014). Persistent Depression after Cardiac Surgery: A Three Months Follow-Up Study. Psychosomatics, 55, 261-271. https://doi.org/10.1016/j.psym.2013.12.011

Pérez, H. (2007). Anxiety and Concerns of the Child and Adolescent before Surgery. Specialty Thesis, Mexico City: UNAM.

Pirraglia, P., Peterson, J., Williams, P., Gorki, L., \& Charlson, M. (1999). Depressive Symptomatology in Coronary Artery Bypass Graft Surgery Patients. International Journal of Geriatric Psychiatry, 14, 668-680. https://doi.org/10.1002/(SICI)1099-1166(199908)14:8<668::AID-GPS988>3.0.CO;2-9

Ramos, B., Figueroa, C., Alcocer, L., \& Rincon, S. (2011). Validation of the Quality of Life Questionnaire Related to Health in Patients with Heart Failure. In-Keys of Thought, 5, 173-189.

Rodríguez, G. (2000). Preparation of Patients for Invasive and Surgical Medical Procedures. Cognitive and Behavioral Aspects. Mexico: Miguel Ángel Porrúa.

Rothenhäusler, H., Ehrentraut, S., Kapfhammer, H., Lang, C., Zachoval, R., Bilzer, M., Schelling, G., \& Gerbes, A. L. (2002). Psychiatric and Psychosocial Outcome of Orthotopic Liver Transplantation. Psychotherapy and Psychosomatics, 5, 285-297. https://doi.org/10.1159/000064811

Rozanski, A., Blumenthal, J. A., Davidson, K. W., Saab, P. G., \& Kubzansky, L. (2005). The Epidemiology, Pathophysiology and Management of Psychosocial Risk Factors in Cardiac Practice: The Emerging Field of Behavioral Cardiology. Journal of the American College of Cardiology, 45, 637-651.

https://doi.org/10.1016/j.jacc.2004.12.005

Sánchez-Román, S., Velasco-Furlong, L., Rodríguez-Carvajal, R., \& Baranda-Sepúlveda, J. (2006). Efficacy of a Cognitive-Behavioral Program for the Reduction of Anxiety in Medically Ill Patients. Revista de Investigacion Clinica, 58, 540-546.

Sandrino, V. L., Meléndez, E. H., Mederosm, D. H., \& Carracedo, I. C. (2016). Sociodemographic Characteristics and Negative Emotional States in Patients with Cardiovascular Disease with Surgical Indication. Revista de Enfermedades Cardiovasculares, 8, 164-172.

Snaith, R. P. (2003). The Hospital to Anxiety and Depression Scale. Health and Quality of Life Outcomes, 1, 1-4. https://doi.org/10.1186/1477-7525-1-29

Sobieralska, K., Rydygier, L., Copernicus, P., Copernicus, N., Michalak, M., Wielki, K. et al. (2015). Stress Associated with Orthopedic Surgery and Feeling Pain. In A. Rosiek, \& K. Leksowski (Eds.), Organizational, Culture and Ethics in Modern Medicine (pp. 372-394). Poland: Medical Information Science.

Soler, E., Faus, M. T., Montaner, M. C., Faus, R., Morales, F., \& Martínez-Pons, C. (1999). Postoperative Pain: Prevalence Characteristics and Pharmacotherapy during the First Day in a Hospital. Pharmacoepidemiology and Drug Safety, 8, 179-188.

Vallejos, J., \& Obergorn, S. (2017). Resilience and Coping Styles in Cardiovascular Patients in a Hospital Es. Salud of Lima. Psyche Magazine, 6, 211-218.

Vilchis, A., Secín, R., López, A., \& Córdova, V. (2010). Frequency of Anxiety and Depres- 
sion in Adult Patients in the Emergency Room of a General Non-Governmental Hospital and Identification by the Emergency Doctor. Medical Internships in Mexico, 26, 5-10.

Zigmond, A. S., \& Snaith, R. P. (1983). The Hospital Anxiety and Depression Scale. Acta Psychiatrica Scandinavica, 67, 361-370.

https://doi.org/10.1111/j.1600-0447.1983.tb09716.x 